\title{
Magnetization transfer contrast MRI in GFP-tagged live bacteria
}

\author{
VALERIA RIGHI $^{1-3}$, MELISSA STARKEY $^{4,5}$, GEORGE DAI $^{2}$, LAURENCE G. RAHME $^{4}$ and ARIAA. TZIKA ${ }^{1,2}$ \\ ${ }^{1}$ NMR Surgical Laboratory, Department of Surgery, Massachusetts General Hospital and Shriners Burns Hospital, \\ Harvard Medical School; ${ }^{2}$ Department of Radiology, Massachusetts General Hospital, Harvard Medical School, \\ Athinoula A. Martinos Center for Biomedical Imaging, Boston, MA 02114, USA; ${ }^{3}$ Department of The Quality of Life Science, \\ University of Bologna, Rimini, RN I-47921, Italy; ${ }^{4}$ Molecular Surgery Laboratory, Department of Surgery, \\ Massachusetts General Hospital and Shriners Burns Institute, Harvard Medical School, Boston, MA 02114; \\ ${ }^{5}$ American College of Physicians, Philadelphia, PA 19106, USA
}

Received December 28, 2017; Accepted May 22, 2018

DOI: $10.3892 / \mathrm{mmr} .2018 .9669$

\begin{abstract}
Green fluorescent protein (GFP) is a widely utilized molecular reporter of gene expression. However, its use in in vivo imaging has been restricted to transparent tissue mainly due to the tissue penetrance limitation of optical imaging. Magnetization transfer contrast (MTC) is a magnetic resonance imaging (MRI) methodology currently utilized to detect macromolecule changes such as decrease in myelin and increase in collagen content. MTC MRI imaging was performed to detect GFP in both in vitro cells and in an in vivo mouse model to determine if MTC imaging could be used to detect infection from Pseudomonas aeruginosa in murine tissues. It was demonstrated that the approach produces values that are protein specific and concentration dependent. This method provides a valuable, non-invasive imaging tool to study the impact of novel antibacterial therapeutics on bacterial proliferation and perhaps viability within the host system, and could potentially suggest the modulation of bacterial gene expression within the host when exposed to such compounds.
\end{abstract}

\section{Introduction}

Pseudomonas aeruginosa ( $P$. aeruginosa) is one of the ESKAPE bacterial species that is particularly concerning, because they represent the largest group of nosocomial pathogens with growing incidences of antibiotic resistance $(1,2)$. Plethora of studies are focused on eliminating or reducing $P$. aeruginosa infection by using novel molecules (3-5). The main problem is tracking the action of these molecules

Correspondence to: Dr Aria A. Tzika, NMR Surgical Laboratory, Department of Surgery, Massachusetts General and Shriners Burns Hospitals, Harvard Medical School, 51 Blossom Street, Room 261, Boston, MA 02114, USA

E-mail: atzika@hms.harvard.edu

Key words: magnetization transfer contrast imaging, green fluorescent protein, Pseudomonas aeruginosa, burn mouse model, in vitro cells, in vivo mouse in vivo, with a non-invasive method. Magnetic resonance imaging (MRI) can be considered as a non-invasive method for monitoring the course of the infection. An MRI approach using USPIO nanoparticles as molecules to label the macrophages that are present in the infected area has been reported and seems to open perspectives for testing novel anti-infective compounds (6,7). Recently, the explosion in available fluorescent proteins promises a wide variety of new tools for biological imaging, and in particular, for protein labeling and cell tracing (8). In the last decade, Green Fluorescent Protein (GFP) has been frequently used as a marker of gene expression, since it is non-toxic for both animals and bacteria and thus useful for in vivo imaging (9). The introduction of GFP has revolutionized the field of cell biology and fluorescence microscopy (10). GFP is a naturally fluorescent protein, consisting of an 11-strand $\beta$-barrel wrapped around a central helix that is widely utilized as a fluorescent marker of gene expression (11). GFP is detected by optical spectroscopy through its fluorescent properties; the protein has a major excitation peak at $395 \mathrm{~nm}$ and in a normal solution gives emission peaking at $508 \mathrm{~nm}$ (11). In vivo GFP can be detected only if the tissue is transparent or if protein expression is close to tissue surface (12). Due to this limitation, most experiments utilizing this marker are focused on in vitro cell cultures, ex vivo histology slices, or transparent animal models $(13,14)$.

GFP is extensively used in animal models, in transplantation studies to determine the fate of transplanted cells, as well as for studying various biological processes. Published studies on in vivo MRI using GFP protein as a marker to label tumor cells for example melanoma cells (15) or stem cells (16) suggested that the labeling does not affect expression of other genes.

Recently, the magnetization transfer contrast (MTC) technique was used to detect GFP and was shown to produce protein-specific values that seemed to be concentration dependent (17). MTC MRI has been utilized for detecting early macromolecular changes in the $\mathrm{Tg} 2576$ mouse model of Alzheimer's disease (18) for localizing the signal to noise ratio (SN) in vivo (19). MTC is an MRI technique able to detect changes in macromolecule concentration and composition (20). MTC is commonly used to track changes in myelination as a 
way to grade multiple sclerosis lesions (21). Recently, MTC has also been utilized to detect macromolecular accumulation in a mouse model of early Alzheimer's disease (18).

The MTC technique uses the application of a radiofrequency pulse at a specific distance from the water resonance, known as the offset frequency. This radiofrequency pulse causes a loss of signal intensity proportional to macromolecular concentration. When combined with a reference image, where the radiofrequency pulse is not applied, the percent of signal loss can be quantified in what is referred to as the magnetization transfer ratio (MTR). Specifically, MTC evaluates changes in semisolid macromolecules (22).

This provides a flexible, non-invasive in vivo molecular imaging system exclusively dependent on the concentration of the fluorescent reporter. Starting from these results the idea of this work was the possibility to follow the P. aeruginosa infection in vivo using the MTC MRI method and the GFP as a molecular marker.

\section{Materials and methods}

Bacterial strains and growth conditions. UCBPP-PA14 (PA14) is a $P$. aeruginosa human clinical isolate (23). GFP-tagged $P$. aeruginosa (PA-GFP), (Fig. 1), GFP-tagged E. coli ( $E C$-GFP) (both tagged with a stable plasmid expressing GFP) and non-fluorescent $P$. aeruginosa $(P A)$ cells were grown overnight in $5 \mathrm{ml}$ LB Lenox medium (Thermo Fisher Scientific, Inc., Waltham, MA, USA) at $37^{\circ} \mathrm{C}$ under $200 \mathrm{rpm}$ orbital shaking using glass tubes (VWR). The next day, bacteria were centrifuged, re-suspended and diluted in phosphate-buffered saline (PBS) to final concentrations of $5 \times 10^{6}$ and $5 \times 10^{5}$ cells $/ \mathrm{ml}$. The latter concentration is equivalent to the $P A$ inoculum used in a murine burn and infection model. Then $0.2 \mathrm{ml}$ microfuge tubes were filled to the maximum capacity with the diluted cultures (23). Fluorescence of the cells was confirmed by microscopy under the 1st section talking about the bacteria.

Phantom. One phantom was prepared to test and calibrate the experiments. Three microfuge tubes (capacity $0.2 \mathrm{ml}$ ) were filled to the maximum capacity with the diluted cultures of $P A$-GFP, EC-GFP and $P A$ cells, respectively, and placed in a Falcon tube $(2.7 \mathrm{~cm}$ inner diameter) filled with isotonic saline $(\mathrm{NaCl})$ solution.

Animals. Six weeks old, CD-1 mice were anesthetized and a leg burn injury of 5\% total burn surface area (TBSA) was produced on the right thigh muscle. Briefly, animals were anesthetized with Xylazine $(13 \mathrm{mg} / \mathrm{kg}$, i.p.) and Ketamine ( $87 \mathrm{mg} / \mathrm{kg}$, i.p.), thermally injured (5-8\% of body surface) on the shaved abdomen dermis, and intra-dermally infected into the burn eschar. Mice were randomized into one experimental and control groups ( $\mathrm{N}=6$ per group). The experimental group consisted of burned mice infected with $P A$-GFP-tagged PA14 $P$. aeruginosa strain containing no plasmid and the control group consisted of burned mice infected with wild-type P. aeruginosa. Mice were infected as described in Rahme et al (23). An inoculum of $5 \times 10^{5}$ PA14 cells in $100 \mu \mathrm{l}$ of PBS was injected intradermal into the burn eschar. The animal protocol was approved by the Massachusetts General Hospital Institutional Animal Care and Use Committee.
MRI experiments. The mice were imaged $12 \mathrm{~h}$ post-burn and infection. During MRI, mice were kept anesthetized with a mixture of isoflurane and maintained at $37^{\circ} \mathrm{C}$.

We used a triple phantom in a $4.7 \mathrm{~T}$ horizontal bore magnet (20 $\mathrm{cm}$ bore diameter, Magnex Scientific, using a Bruker Avance console). The images were acquired in a $4.7 \mathrm{~T}$ horizontal magnet, $20 \mathrm{~cm}$ bore, equipped with gradient system capable of $39 \mathrm{G} / \mathrm{cm}$, Magnex Scientific, using a Bruker Avance console (Bruker BioSpin, Billerica, MA, USA) with a custom-built volume coil of $3 \mathrm{~cm}$ inner diameter and $10 \mathrm{~cm}$ active length. The main magnetic field (B0) was shimmed and the RF filed (B1) was calibrated. We acquired a RARE sequence (also known as Fast Spin Echo, FSE) with magnetization transfer (24-26). The imaging pulse sequence comprised a pre-saturation pulse at the designated offset frequency followed by a spin echo sequence with TE/TR=7.95/2,000 msec. Images were recorded with a $128 \times 128$ matrix, field of view, $3 \times 3 \mathrm{~cm}$; slice thickness, $3 \mathrm{~mm}$; and average, 1 . Pre-saturation off-resonance pulses ranged from \pm 0.05 to $\pm 0.4 \mathrm{kHz}$.

Magnetization transfer ratios (MTR) in the form of MTR=(unsaturated-saturated)/unsaturated were calculated from the signal intensities of regions of interest (ROI) using Paravision software (Bruker BioSpin).

Statistical analysis. Data are expressed as a mean \pm standard deviation. Comparisons between groups were performed in each group using a two-tailed t-test $(\mathrm{P}$-value $=0.05)$. Moreover we implemented the analysis with a One-Way ANOVA analysis followed by Tukey's post hoc test using the Metaboanalyst online softaware (http://www.metaboanalyst.ca/).

\section{Results}

GFP cell model. We compared the MTC profiles of GFP-tagged $P$. aeruginosa cells to those of the non-fluorescent $P$. aeruginosa, GFP-tagged and $E$. coli cells in culture. Cells were visualized in $0.2 \mathrm{ml}$ tubes filled to capacity with $5 \times 10^{5}$ cells $/ \mathrm{ml}$. The non-fluorescent $P$. aeruginosa, was chosen as a non-specific control to compare against GFP-tagged bacteria, whereas E. coli was used as a specific control for the GFP to compare with both tagged and wild-type $P$. aeruginosa. The goal was to find the frequency at which there was the largest difference between GFP-tagged P. aeruginosa and E. coli, and non-fluorescent $P$. aeruginosa (Fig. 2). Samples were imaged first without and then with MTC. Nine MTC datasets were acquired from 0.05 to $0.4 \mathrm{kHz}$. The MTR was calculated from the images, and is shown in Fig. 3A. We found the largest difference between $0.2,0.25$ and $0.3 \mathrm{kHz}$ (the peak difference was at $0.25 \mathrm{kHz}$ ) for $E C$-GFP and for $P A$-GFP with respect to PA (Fig. 3A). Pseudo-colored pixel by pixel MTR calculations visually show a clear difference between the $P A$-GFP and $P A$ phantoms (Fig. 3B); we found a statistically significant difference between $P A$ and $E C$-GFP $(\mathrm{P}<0.0001)$, and an even greater statistically significant difference between $P A$ and $P A$-GFP $(\mathrm{P}=0.00001)$. These simple t-test analyst is confirmed by using a multiple comparison Tukey's analysis (One-way ANOVA Analysis). The PA samples are strongly different from $E C$-GFP and $P A$-GFP and the data are statistically significant with $\mathrm{P}<0.001$ and $\mathrm{P}<0.01$ respectively. 


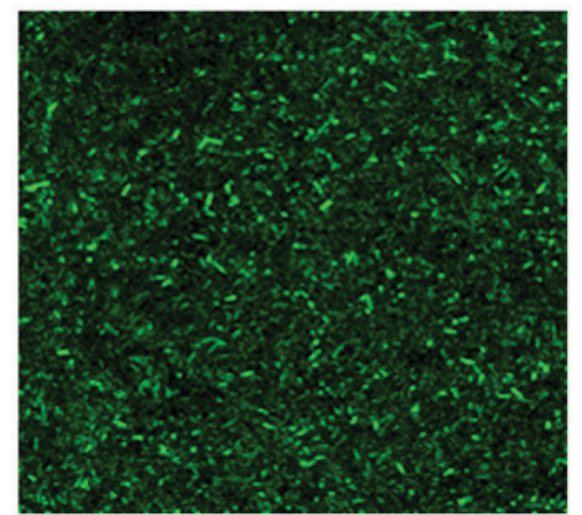

Figure 1. GFP-tagged Pseudomonas aeruginosa cells viewed at x600 magnification. GFP, green fluorescent protein.

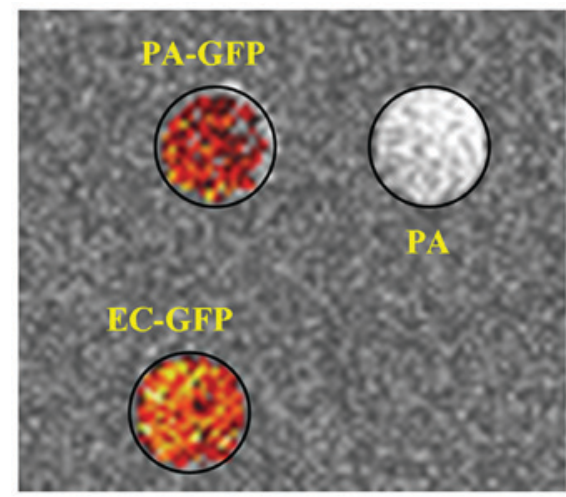

Figure 2. Pseudocolored pixel of $E C$-GFP, $P A$-GFP and PA at $0.25 \mathrm{kHz}$ offset. GFP, green fluorescent protein; EC-GFP, GFP-tagged E. coli; PA-GFP, GFP-tagged Pseudomonas aeruginosa.

GFP mouse model. The comparison of the GFP-tagged and non-tagged live $P$. aeruginos $a$ and $E$. coli cells using MTC MRI indicated that this method was sensitive enough to distinguish between GFP-tagged and non-tagged bacteria at cell concentrations relevant to those to be used in animal infection models. Accordingly, the MTC MRI profiles of mice infected with non-tagged $P$. aeruginosa and mice infected with GFP-tagged $P$. aeruginosa were compared to determine the frequency with the largest difference between them in the infected a burn area. Fig. 4 shows the in vivo MRI results from our experiments in mice, and the MTR maps demonstrated an enhancement in the GFP expressing infected animals. The two groups (GFP-positive and GFP-negative) were imaged first without and then with MTC. In addition, nine MTC datasets were acquired from 0.2 to $1.6 \mathrm{kHz}$. The control animal did not give any appreciable signal at this setting. The MTR was calculated from the images, and is shown in Fig. 5A. We found the largest difference between $0.8,1$ and $1.2 \mathrm{kHz}$, the peak difference being at $1 \mathrm{kHz}$, for the GFP-positive with respect to the GFP-negative mice. Fig. 5B shows the calculated MTR values; we found a statistically significant difference between $\mathrm{GFP}^{+}$and $\mathrm{GFP}^{-}$with a $\mathrm{P}<0.0001$.

The visual representation is more useful to assess the spatial distribution of signal changes compared to a single ROI analysis. The unsaturated images for a representative GFP expressing and control animal demonstrated different signal intensities. The MTR maps demonstrated an enhancement in the GFP expressing animals.

\section{Discussion}

MTC MRI methods have been used before to distinguish intrinsic macromolecule concentration changes. The main advantages of detecting GFP, an extrinsic protein marker, with MTC MRI compared to other MRI based reporters, such as USPIO are the non-toxicity of the protein and the potential to detect the expression of a specific gene or product in vivo serially and non-invasively. Our results confirm that we can detect GFP-tagged live bacteria using MTC MRI both in vitro and in vivo.

Comparison of the GFP-tagged and non-tagged live $P$. aeruginosa and $E$. coli cells using the MTC MRI methodology indicated that this method was sensitive enough to distinguish between GFP-tagged and non-tagged bacteria and to successfully image in vivo GFP-tagged $P$. aeruginosa in a non-invasive manner. We observed a difference in the MRI MTR value between $P A$ and $P A G F P$, which was statistically significant $(\mathrm{P}<0.0001)$.

Also, we report an in vivo study of GFP-tagged MTC MRI in a burn mouse model infected with $P$. aeruginosa. The utility of this method is to visualize bacterial infections in vivo in real time, and to study the impact of novel therapeutics on bacterial proliferation and viability within the host system. The use of an extrinsic protein marker provides an added flexibility. The main advantages of detecting GFP with MTC MRI over other MRI based reporters includes that there are multiple GFP mouse lines available and it poses no toxicity to the host or the bacteria.

Our results confirm the hypothesis that we can detect GFP-tagged live bacteria using MTC MRI.

The study reported here assessed detection of GFP through MTC MRI both in vitro and in vivo experiments. To provide optimal results and the best off-set frequency we worked on the fine-tuned and at the end we found that $1 \mathrm{kHz}$ offset as the most robust offset frequency for the MTC detection of GFP and provided the difference between tagged and non-tagged mice. Our results are similar to the data reported by Pérez-Torres et al (17).

We were able to successfully use this methodology to image in vivo GFP-tagged $P$. aeruginosa in a murine burn and infection model, showing the utility of MTC for tracking bacterial proliferation and gene expression in vivo in animal models in a non-invasive manner. The significance of this method is that it can be used to visualize bacterial infections in vivo in real time without being restricted to the use of transparent tissue necessary for optical imaging. The innocuous nature of the technology allows for repeated imaging over time without damage to the host or the bacteria. Furthermore, this in vivo, MRI molecular imaging technique can detect varying levels of the GFP reporter, further establishing its utility for studying host-bacterial interactions. In addition to the visualizing bacterial infections and expression of GFP tagged gene, in vivo and in real time, this method could be suitable for assessing the efficacy of novel therapeutics on specific targets, and, bacterial proliferation and viability within the host system.

Overall, this method provides a valuable, non-invasive imaging tool to study the impact of novel antibacterial 

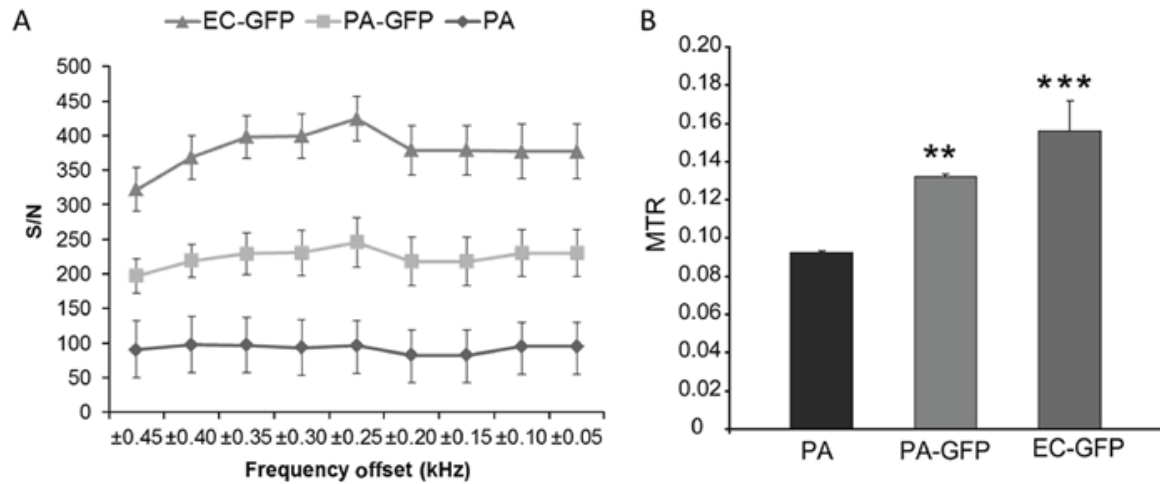

Figure 3. (A) Region based MTR calculations for the different frequency offsets for $P A, P A$-GFP and $E C$-GFP from 0.05 to $0.45 \mathrm{kHz}$ (frequency offset) (B) MTR (mean \pm SE) of $P A, P A$-GFP and $E C$-GFP. Data were analyzed using one-way ANOVA analysis followed by Tukey's post hoc test. ${ }^{* *} \mathrm{P}<0.01$ and ${ }^{* * * *} \mathrm{P}<0.001$ vs. PA. PA, Pseudomonas aeruginosa; GFP, green fluorescent protein; EC-GFP, GFP-tagged E. coli; PA-GFP, GFP-tagged Pseudomonas aeruginosa; MTR, magnetization transfer ratio.

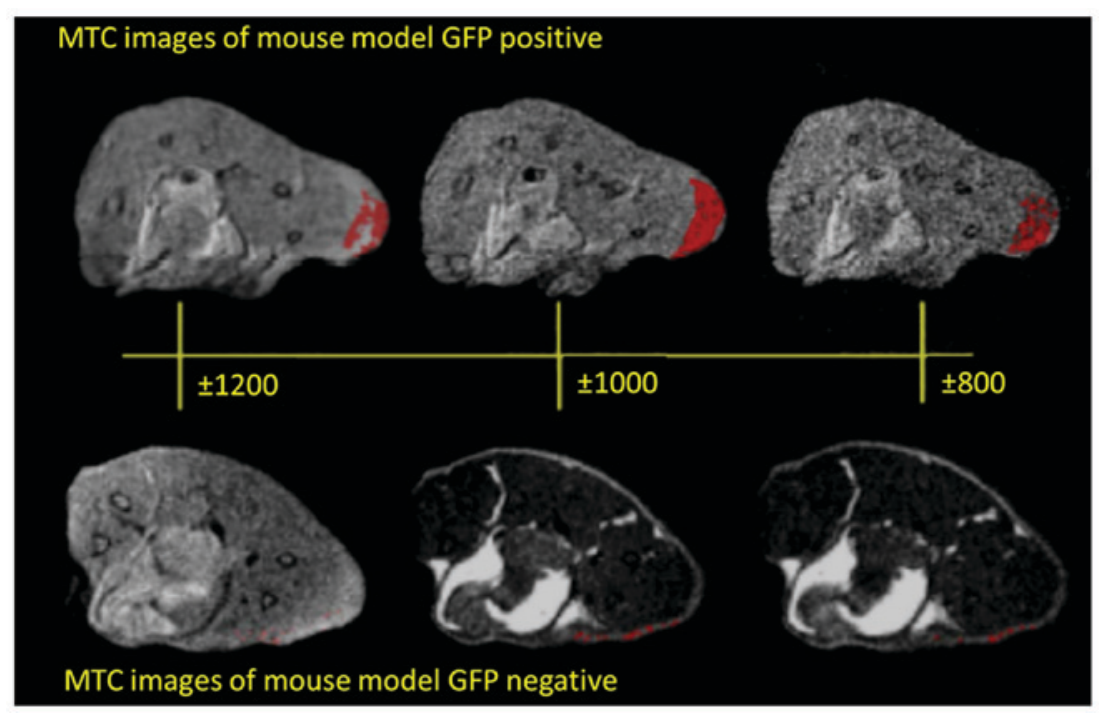

Figure 4. Pseudocolored pixel of $\mathrm{GFP}^{+}$(upper) and GFP- (lower) mice MR images at $1 \mathrm{kHz}$ offset. GFP, green fluorescent protein.
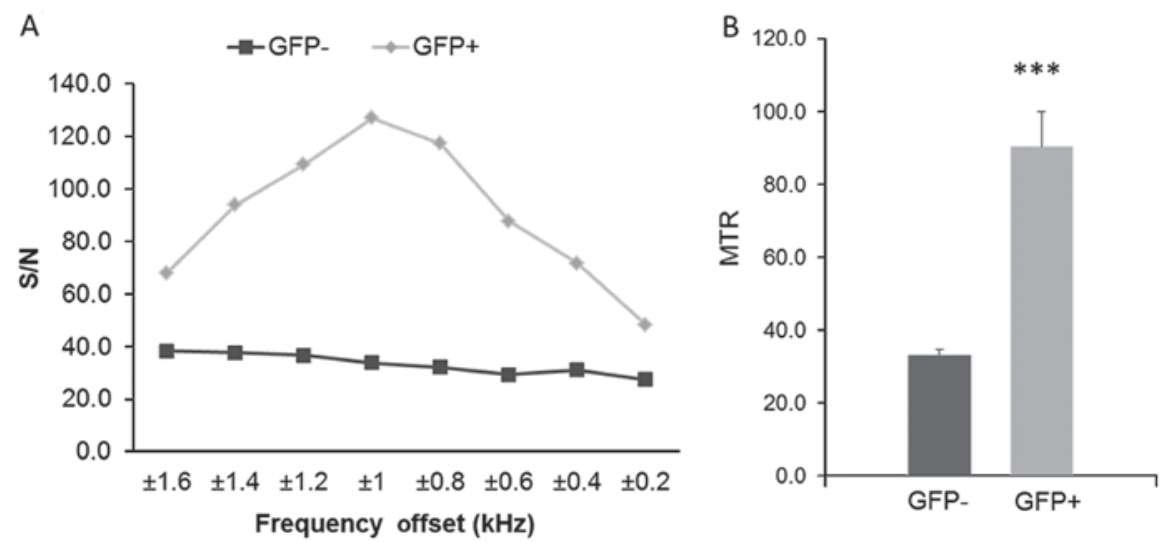

Figure 5. (A) Region based MTR calculations for the different frequency offsets for mice GFP- and mice $\mathrm{GFP}^{+}$from 0.2 to $1.6 \mathrm{kHz}$ (frequency offset). (B) MTR (mean $\pm \mathrm{SE}$ ) of GFP' and $\mathrm{GFP}^{+}$, P-values. ${ }^{* * *} \mathrm{P}<0.0001$ vs. GFP. GFP, green fluorescent protein; MTR, magnetization transfer ratio.

therapeutics on bacterial proliferation and perhaps viability within the host system, and could potentially give clues to the modulation of bacterial gene expression within the host when exposed to such compounds.

\section{Acknowledgements}

This abstract was presented at the International Society of Magnetic Resonance Annual Meeting hold in Stockholm in 2010 
and was published as Abstract no. 5135 Proceeding 'Righi V, Starkey M, Dai G, Rahme LG and Tzika AA: Magnetization transfer contrast MRI in GFP-tagged live bacteria. Proc Intl Soc Mag Reson Med 18: 513, 2010. ISBN: 978-1-61782-008-3'. This abstract was presented at the International Society of Magnetic Resonance Annual Meeting hold in Montreal in 2011 and was published as Abstract no. 662 Proceeding 'Righi V, Starkey M, Rahme LG, Tompkins RG and Tzika AA: Magnetization Transfer Contrast MRI detects Pseudomonas Aeruginosa bacterial infection a mouse burn model. Proc Intl Soc Mag Reson Med 19: 662,2011. ISBN: 978-1-61839-284-8. ISSN: 1545-4428'.

\section{Funding}

The present study was funded by Shriners Burn Hospitals and NIH grants (NIH grant no. R01AI134857 and grant no. R33AI105902).

\section{Availability of data and materials}

All data generated or analysed during this study are included in this published article.

\section{Authors' contributions}

VR analyzed the samples and interpreted data and wrote the manuscript; MS prepared the samples and helped in writing the manuscript; GD helped in the MRI acquisition data; LR supported the study, helped in writing the manuscript and interpreting the results, and organized the cells and mice preparation, AT designed the study, helped in data interpretation and wrote the manuscript. All authors read and approved the final manuscript.

\section{Ethics approval and consent to participate}

This study was carried out in strict accordance with the recommendations of the Guide for the Care and Use of Laboratory Animals of the National Institutes of Health. The protocol was approved by the Committee on the Ethics of Animal Experiments at Massachusetts General Hospital (Permit no. 2006N000093/2).

\section{Patient consent for publication}

Not applicable.

\section{Competing interests}

The authors declare that they have no competing interests.

\section{References}

1. Turner KH, Everett J, Trivedi U, Rumbaugh KP and Whiteley M Requirements for Pseudomonas aeruginosa acute burn and chronic surgical wound infection. PLoS Genet 10: e1004518, 2014.

2. Pendleton JN, Gorman SP and Gilmore BF: Clinical relevance of the ESKAPE pathogens. Expert Rev Anti Infect Ther 11: 297-308, 2013
3. Church D, Elsayed S, Reid O, Winston B and Lindsay R: Burn wound infections. Clin Microbiol Rev 19: 403-434, 2006.

4. Lyczak JB, Cannon CL and Pier GB: Establishment of Pseudomonas aeruginosa infection: Lessons from a versatile opportunist. Microbes Infect 2: 1051-1060, 2000.

5. McVay CS, Velásquez M and Fralick JA: Phage therapy of Pseudomonas aeruginosa infection in a mouse burn wound model. Antimicrob Agents Chemother 51: 1934-1938, 2007.

6. Andronesi OC, Mintzopoulos D, Righi D, Psychogios N Kesarwani M, He J, Yasuhara S, Dai G, Rahme LG and Tzika AA: Combined off-resonance imaging and $\mathrm{T} 2$ relaxation in the rotating frame for positive contrast MR imaging of infection in a murine burn model. J Magn Reson Imaging 32: 1172-1183, 2010.

7. Starkey M, Lepine F, Maura D, Bandyopadhaya A, Lesic B, He J Kitao T, Righi V, Milot S, Tzika A and Rahme L: Identification of anti-virulence compounds that disrupt quorum-sensing regulated acute and persistent pathogenicity. PLoS Pathog 10: e1004321, 2014.

8. Shaner NC, Steinbach PA and Tsien RY: A guide to choosing fluorescent proteins. Nat Methods 2: 905-909, 2005.

9. Chalfie M, Tu Y, Euskirchen G, Ward WW and Prasher DC: Green fluorescent protein as a marker for gene expression. Science 263: 802-805, 1994.

10. Stepanenko OV, Verkhusha VV, Kuznetsova IM, Uversky VN and Turoverov KK: Fluorescent proteins as biomarkers and biosensors: Throwing color lights on molecular and cellular processes. Curr Protein Pept Sci 9: 338-369, 2008.

11. Tsien RY: The green fluorescent protein. Annu Rev Biochem 67: 509-544, 1998

12. Helmchen F and Denk W: Deep tissue two-photon microscopy. Nat Methods 2: 932-940, 2005

13. Shaner NC, Patterson GH and Davidson MW: Advances in fluorescent protein technology. J Cell Sci 120: 4247-4260, 2007.

14. Zhang J, Campbell RE, Ting AY and Tsien RY: Creating new fluorescent probes for cell biology. Nat Rev Mol Cell Biol 3: 906-918, 2002.

15. WunderbaldingerP,Josephson L,BremerC,Moore A, Weissleder R: Detection of lymph node metastases by contrast-enhanced MRI in an experimental model. Magn Reson Med 47: 292-297, 2002.

16. Pawelczyk E, Jordan EK, Balakumaran A, Chaudhry A, Gormley N, Smith M, Lewis BK, Childs R, Robey PG and Frank JA: In vivo transfer of intracellular labels from locally implanted bone marrow stromal cells to resident tissue macrophages. PLoS One 4: e6712, 2009.

17. Pérez-Torres CJ, Massaad CA, Hilsenbeck SG, Serrano F and Pautler RG: In vitro and in vivo magnetic resonance imaging (MRI) detection of GFP through magnetization transfer contrast (MTC). Neuroimage 50: 375-382, 2010.

18. Pérez-Torres CJ, Reynolds JO and Pautler RG: Use of magnetization transfer contrast MRI to detect early molecular pathology in Alzheimer's disease. Magn Reson Med 71: 333-338, 2014

19. Bolding MS, Reid MA, Avsar KB, Roberts RC, Gamlin PD, Gawne TJ, White DM, den Hollander JA and Lahti AC: Magnetic transfer contrast accurately localizes substantia nigra confirmed by histology. Biol Psychiatry 73: 289-294, 2013.

20. Wolff SD and Balaban RS: Magnetization transfer contrast (MTC) and tissue water proton relaxation in vivo. Magn Reson Med 10: 135-144, 1989.

21. Horsfield MA: Magnetization transfer imaging in multiple sclerosis. J Neuroimaging 15 (Suppl 4): 58S-67S, 2005.

22. Henkelman RM, Stanisz GJ and Graham SJ. Magnetization transfer in MRI: A review. NMR Biomed 14: 57-64, 2001.

23. Rahme LG, Stevens EJ, Wolfort SF, Shao J, Tompkins RG and Ausubel FM: Common virulence factors for bacterial pathogenicity in plants and animals. Science 268: 1899-1902, 1995;

24. Forsén $S$ and Hoffman RA: Study of moderately rapid chemical exchange reactions by means of nuclear magnetic double resonance. J Chem Phys 39: 2892, 1963.

25. Baguet E and Roby C: Off-resonance irradiation effect in steady-state NMR saturation transfer. J Magn Reson 128: 149-160, 1997.

26. Sun PZ, Van Zijl PC and Zhou J: Optimization of the irradiation power in chemical exchange dependent saturation transfer experiments. J Magn Reson 175: 193-200, 2005. 